\title{
ORIGIN OF PERPENDICULAR MAGNETIC ANISOTROPY IN M-O MULTILAYERS (Invited)
}

\author{
G. BAYREUTHER
}

Institete für Angewandte Physik, Universität Regensburg, 93040 Regensburg, Fed. Rep. Germany

\section{Introduction}

Magnetic anisotropy plays a key role in most technical applications of magnetically ordered materials. In particular, in thin films and multilayers for magneto-optic recording a magnetic anisotropy with the effective easy axis perpendicular to the film plane is a necessary prerequisite. Despite its technological importance, only very recently significant progress has been achieved in the understanding of the microscopic mechanisms which lead to the perpendicular magnetic anisotropy (PMA) observed in many ultrathin magnetic films and multilayers. In this paper, after a phenomenological discussion of possible contributions to magnetic anisotropy in films and multilayers some experiments will be discussed in relation to recent theoretical results which for the first time seem to establish a correlation between PMA and the electronic structure. Furthermore, it will be shown that semiphenomenological theories are still indispensable to describe the consequences of structural defects for magnetic anisotropies.

\section{Phenomenological approach.}

In thin films magnetic anisotropies are observed with an energy which is either proportional to the film volume or to the film area. The latter are usually assumed to originate from the interfaces of the film and when expressed as an effective volume anisotropy lead to a contribution proportional to the inverse film thickness.

Usually, three different classes of magnetic anisotropies are distinguished:

- dipolar anisotropy

- magneto-elastic anisotropy

- magneto-crystalline anisotropy

Dipole-dipole interactions produce the well-known shape anisotropy which in thin films of cubic material is a uniaxial anisotropy with the film normal being the hard axis. It is a volume anisotropy and its energy density is written as $\epsilon_{A}=2 \pi M_{S}^{2} \cos ^{2} \Phi$ where $\Phi$ is the angle between the (homogeneous) magnetization and the film normal. For many films this is the dominant term of anisotropy and forces the magnetization to lie in the film plane in the absence of a strong perpendicular field.

However, dipolar fields also result in an interface anisotropy in three different cases: i) the magnetization at the interface is different from the value inside the film. This can be due to modified ground state atomic moments or to non-uniform spinwave excitations; ii) a finite interface roughness always reduces the anisotropy ${ }^{1}$; iii) the discrete nature of the atomic dipoles produces an effective interface anisotropy ${ }^{2}$. This effect which may amount to ca. $10 \%$ of the shape anisotropy will be reduced somewhat if the spin density distribution as given by band calculations is taken into account.

In general, dipolar interface anisotropies have to be considered if the uncertainty of the total anisotropy constant is below $\approx 10 \%$.

Magneto-elastic anisotropy resulting from elastic strain and magneto-elastic coupling is known to significantly contribute to magnetic anisotropy in thin films ${ }^{3}$. Its microscopic origin is spin-orbit coupling. Two different cases have to be distinguished: if the strain is uniform like for perfectly pseudomorphic growth with a certain lattice mismatch this anisotropy is a volume term; above the critical film thickness for the onset of misfit dislocation formation it behaves like an interface anisotropy ${ }^{4}$.

Magneto-crystalline anisotropy is directly related to the symmetry of the system under consideration. In $3 d$ metals it results mainly from spin-orbit coupling. Néel was the first to realize that a uniaxial 
magnetic anisotropy is to be expected due to the broken translational symmetry at surfaces and interfaces. The related interface anisotropy energy depends on the angle $\Phi$ between the magnetization and the film normal and is conveniently expanded in a power series of $\cos \Phi$. Usually, only the lowest order non-vanishing term is considered which is proportional to $\cos ^{2} \Phi$. However, it has been pointed out recently ${ }^{5}$ that the next higher term proportional to $\cos ^{4} \Phi$ may also play an important role.

With Néel's pair energy model and by using empirical magnetostriction data it is possible to predict anisotropy values for different surfaces. Agreement with experimentally observed interface anisotropies is sometimes rather good, but sometimes poor. As a consequence, the validity of Néel's model has been questioned by several authors. In some cases the restriction to nearest neighbour interactions is responsible for observed discrepancies. In addition, it must be emphasized that elementary anisotropy constants in this model are merely phenomenological constants and their actual value cannot be derived neither by sign nor by magnitude within Néel's theory. On the other hand, it is clear from its basic concept that the interface anisotropy energy is determined both by the magnetic and the nonmagnetic material forming the interface. A consistent description can only be expected from a microscopic theory.

Microscopic approach.

The fundamental understanding that magnetic interface anisotropy results from electronic interactions at the interface is based on experiments where a given ferromagnetic material with a given surface orientation, e.g. $\mathrm{Fe}(110)$ or $\mathrm{Co}(0001)$, is covered by different non-magnetic metals and the change in magnetic anisotropy is measured. The following values of single interface anisotropy constants, $K_{S}$, have been reported (note that in agreement with Néel's convention a positive value means an easy plane and a negative value a perpendicular easy axis): for $\mathrm{Fe}(110) / \mathrm{Au}(111) K_{S}=-.69 \mathrm{erg} / \mathrm{cm}^{2}$ (or $\left.\mathrm{mJ} / \mathrm{m}^{2}\right)^{6}$ or $-.72 \mathrm{erg} / \mathrm{cm}^{2} 7$; negative values between -.12 and $-.97 \mathrm{erg} / \mathrm{cm}^{2}$ for $\mathrm{Fe}(110) / \mathrm{Cr}, \mathrm{Fe}(110) / \mathrm{Cu}, \mathrm{Fe}(110) / \mathrm{Ag}$ and $\mathrm{Fe}(110) / \mathrm{UHV}^{7}$ and a strong easy-plane anisotropy for $\mathrm{Fe}(110) / \mathrm{W}(110)^{7}$ with $K_{S}=1.92 \mathrm{erg} / \mathrm{cm}^{2}$. For Co(0001) interfaces similar results have been reported with easy perpendicular axis in most cases (Co/Au, Co/Pd, Co/Pt, $\mathrm{Co} / \mathrm{Cu}, \mathrm{Co} / \mathrm{Ag}$ ) and with easy plane for $\mathrm{Co} / \mathrm{UHV}^{8,9}$.

These findings together with the recent observation of a non-monotonic dependence of $K_{S}$ on the thickness of the non-magnetic overlayer underline the necessity to discuss magnetic interface anisotropy on the basis of electronic band structure which is specific for each film system. Recent progress in $a b$ initio calculations of anisotropy in films and multilayers together with existing controversies will be reviewed.

Influence of defects.

Finally, it is pointed out that the influence of defects such as atomic steps or interface roughness still requires semi-phenomenological theories. As an example, it is shown that the modification of interface anisotropy in $\mathrm{Fe} / \mathrm{Au}(111)$ multilayers by variation of substrate roughness, controlled intermixing at the interfaces and back-diffusion during annealing can be understood on the basis of Néel's model.

Support by the Deutsche Forschungsgemeinschaft is gratefully acknowledged.

1 P.Bruno, J.Phys. F 18 (1988) 129

${ }^{2}$ H.J.G. Draaisma et al., J.Appl. Phys. 64 (1988) 3610

3 F.J.A. den Broeder et al., J.Magnet.Magnet.Mat. 93 (1991) 561

${ }^{4}$ C.Chappert and P.Bruno, J.Appl.Phys. 64 (1988) 5736

${ }^{5}$ H. Fritzsche et al., Phys. Rev. B 49 (1994) ?

${ }^{6}$ G.Lugert and G.Bayreuther, Thin Solid Films 175 (1989) 311

7 H.Fritzsche et al., J.Magnet.Magnet.Mat. 135 (1994) ?

8 P.Beauvillain et al., J.Magnet.Magnet.Mat. 121 (1993) 503 and Phys. Rev. B (1994)?

9 J.Kohlhepp et al., to be published 